\title{
Saúde e desenvolvimento na África Subsaariana: uma reflexão com enfoque em Moçambique
}

I ${ }^{1}$ Fernando Mitano, ${ }^{2}$ Carla Aparecida Arena Ventura, ${ }^{3}$ Pedro Fredemir Palha I

Resumo: O reconhecimento de que a melhoria da saúde da população depende do avanço das condições socioeconômicas é cada vez mais visível nos últimos anos, incentivando mudanças nas políticas nacionais e internacionais. Contudo, em vários países da África Subsaariana, se não todos, essa relação é inversamente proporcional. Assim, com este texto pretende-se refletir sobre a conexão existente entre saúde e desenvolvimento em Moçambique. Esta reflexão oferece uma compreensão sobre o crescimento econômico que se registra no país, porém com maior parte da população a viver em precárias condições e com uma multiplicidade de doenças evitáveis, demonstrando, também, que as políticas adotadas pelo país ainda não favorecem um caminhar conjunto da saúde e desenvolvimento. Essa situação requer que o país aplique políticas de redistribuição de renda e de atenção à saúde em populações mais carentes.

> Palavras-chave: saúde; desenvolvimento; pobreza.

\author{
1 Faculdade de Ciências de \\ Saúde, Universidade Lúrio, \\ Moçambique (piqinamita@ \\ gmail.com). \\ 2 Escola de Enfermagem de \\ Ribeirão Preto, Universidade de \\ São Paulo. Ribeirão Preto-SP, \\ Brasil (caaventu@eerp.usp.br). \\ ${ }^{3}$ Escola de Enfermagem de \\ Ribeirão Preto, Universidade de \\ São Paulo, Ribeirão Preto-SP \\ (palha@eerp.usp.br).
}

Recebido em: 14/10/2014 Aprovado em: 18/01/2016 


\section{Introdução}

A África Subsaariana tem enfrentado dificuldades para se desenvolver, representando uma região do mundo com sérios problemas de saúde pública: maior número de casos de HIV/Aids (LAU; MUULA, 2004) e tuberculose, sendo que três países, África do Sul, Zimbábue e Moçambique, ocupam as três primeiras posições, respectivamente, da classificação dos 22 países que mais contribuem com a tuberculose no globo (WHO, 2015). Para além disso, a região continua a apresentar elevados índices de mortalidade infantil e materna e de doenças infecciosas, que demonstram e contribuem para o baixo índice de desenvolvimento humano. Ainda, mais de 50\% de seus habitantes sofrem de doenças evitáveis provenientes da má qualidade da água, como a cólera e diarreia infantil. Como consequência, a cada 30 segundos, um óbito infantil é registrado devido à malária (ANDRADE, et al., 2012). Embora a pobreza tenha sido reduzida de 1990 a 2015 na região subsaariana, aproximadamente $41 \%$ de sua população ainda vive em extrema pobreza, ou seja, com renda abaixo de 1,25 dólares por dia (UN, 2015), revelando um grave problema de desigualdade social.

Wilson-Strydom e Fongwa (2012) descreveram que, nos últimos anos, os países da África Subsaariana foram afetados por problemas na gestão e administração pública ou estão a se recuperar de conflitos políticos e até de guerras, agravadas por crises nos diversos programas de ajustamentos estruturais, levando os governos a adotar uma postura defensiva em termos de objetivos de desenvolvimento econômico e social, o que se reflete na educação e saúde. Nesse sentido, Appleton, Hoddintt e MacKinnon (1996) revelam que, na África Subsaariana, todos os indicadores de educação e saúde encontravam-se em último lugar. Atualmente, após mais de 18 anos da publicação desses dados, a região continua a ocupar a mesma posição, segundo o relatório dos Objetivos de Desenvolvimento do Milênio (ODM) (UN, 2015). Apesar desse quadro, deve-se advertir, contudo, que há registro de um ligeiro crescimento econômico de até 5,3\% em 2012 e 5,6\% em 2013, acima das taxas médias do período anterior à crise (WORLD BANK, 2012).

Considerando esse cenário, a questão que se pode levantar é: será que o crescimento registrado se reflete na melhoria das condiçôes de saúde da população? Parece-nos que essa não é uma relação direta de causa e efeito e que essa resposta 
não é tão objetiva, uma vez que se verificam nos dias atuais a piora da saúde e a dificuldade de as pessoas terem acesso aos serviços de saúde naquela região.

Desse modo, um estudo desenvolvido pela Organização Mundial da Saúde (OMS) (2012) sobre os sistemas de saúde em África, percepçôes e perspectivas das comunidades revela que os mesmos são frágeis e apresentam déficit da força de trabalho, prestando precários serviços, informações e produtos médicos. Outro fator que o estudo registra são as diferenças entre os países e dentro deles, salientando-se as desigualdades no acesso e atendimento entre os ricos, geralmente detentores do poder, e os considerados pobres. Nesse contexto, a Comissão para os Determinantes Sociais de Saúde afirma que as forças de ordem sociopolítica e econômica determinam as condições em que as pessoas vivem e morrem e como elas podem acessar os serviços de saúde (OMS, 2010).

Moçambique, que se situa nessa região africana, não foge das características descritas acima. Após a independência do domínio português, em 1975, o país entrou em guerra civil até o ano de 1992. Durante os 16 anos de guerra civil, o Serviço Nacional de Saúde ficou fortemente fragilizado, com pouca cobertura populacional tanto das pessoas que viviam nos espaços urbanos quanto das que residiam nos distritos, agravados pelas dificuldades de acesso geográfico. Após a guerra, o país adotou um sistema político democrático e de economia liberal, que elevou o crescimento econômico em 6\% em 2012, reconhecido pelo Banco Mundial. Em 2013, a economia de Moçambique continuou a ser uma das mais dinâmicas da região, com uma taxa real de crescimento de $7 \%$, sendo previsto um crescimento de 8\% para os anos de 2014 e 2015 (BANCO MUNDIAL, 2013; ALMEIDA-SANTOS; ROFFARELLO; FILIPE, 2014).

Outrossim, apesar desse crescimento economico, Moçambique mostra ser, cada vez mais, um país de grandes desigualdades sociais, onde poucas pessoas têm acesso aos bens e estrutura social, como Sistema Nacional de Saúde (SNS) e outros, gerados pela economia, em detrimento da maioria da população. A maioria de sua população ainda vive abaixo da linha da pobreza, sobretudo aqueles que vivem nas áreas rurais, que constituem a maior parte da população moçambicana (SHENGA; PEDRO; MUENDANE, 2013). Essas ideias são corroboradas pelo Relatório do Desenvolvimento Humano das Nações Unidas, de 2015, que coloca o país em 180 lugar dentre os 188 países avaliados. Assim, Moçambique faz parte dos dez países mais pobres do mundo, e é o 70 país com 
pior índice de Desenvolvimento Humano, ultrapassando até nações que se encontram em conflito armado (PNUD, 2015). Relativamente à saúde, o último Inquérito Demográfico e de Saúde de Moçambique, realizado em 2011, reporta que $48 \%$ dos partos não são assistidos nas unidades sanitárias existentes no país, demonstrando claramente a dificuldade que o Serviço Nacional de Saúde tem de cobrir todas as necessidades do parto. Essa situação torna-se mais gritante para as áreas rurais, em que o número total dos partos assistidos foi de $40 \%$ em 2011, de modo que $60 \%$ de partos continuam não sendo assistidos pelo SNS.

Nesse cenário, este estudo busca refletir sobre a conexão existente entre saúde e desenvolvimento em Moçambique, um país em crescimento econômico, porém com grande desigualdade social, onde a maior parte da população vive em precárias condições de vida, com multiplicidade de doenças evitáveis e onde as políticas públicas e a economia ainda não favorecem um caminhar conjunto entre a saúde e o desenvolvimento.

\section{Compreendendo a conexão entre saúde e desenvolvimento}

A realidade mundial dos dias de hoje mostra que saúde e desenvolvimento são intrinsicamente ligados. Basta recordar a Declaração da OMS de 1946, que define a saúde como "um estado de completo bem-estar físico, mental e social, e não simplesmente a ausência da doença ou enfermidade". Sendo assim, fica claro que o alcance e a manutenção do bem-estar social requerem que as pessoas tenham as condições mínimas para a sua sobrevivência: alimentação, habitação e educação, condições que, se ausentes, podem produzir conflitos internos e intrapsíquicos que favorecem o mal-estar. Para isso, é preciso que as pessoas tenham de algum modo desenvolvimento, sobretudo, socioeconômico.

Quando se fala de desenvolvimento, há várias vertentes a serem pensadas: desenvolvimento econômico, comunitário, social, regional, territorial, sustentável (AKERMAN et al., 2012; ANDRADE et al., 2012; VILANA; ELIAS, 2007; GADELHA et al., 2011; FURTADO, 2000; TISCH; WALLACE, 1994). Seja qual for a vertente polissêmica do desenvolvimento, seu benefício final deveria recair sobre o homem. Entretanto, esse benefício pode não produzir saúde para a maioria se não for entendido como um bem comum. Nesse sentido, se o desenvolvimento for compreendido como propriedade de alguns em detrimento de outros, pode-se justificar a má qualidade de saúde que atinge várias pessoas 
do mundo, sobretudo da África Subsaariana e especificamente de Moçambique.

Idealmente, com o desenvolvimento, espera-se que as pessoas tenham uma vida longa, liberdade, dignidade, justiça e riqueza. Como afirmam Akerman et al. (2012), o desenvolvimento implica a produção do próprio homem e a capacidade de produzir meios e bens que satisfaçam suas necessidades. Portanto, ele deve se refletir na melhoria de infraestrutura física, qualidade de vida da população e oportunidades que possam transformar o próprio homem, ou seja, o homem deve ser capaz de melhorar a sua vida.

Em Moçambique, o desenvolvimento que se verifica no nível econômico caminha em direções e sentidos opostos aos da melhoria de saúde da população. O’Langhlin (2012) defendeu que a saúde das pessoas e a sua qualificação constituem um pré-requisito para o desenvolvimento. Esse autor reconheceu, ainda, que as políticas macroeconômicas aplicadas, sobretudo, em países como Moçambique estimulam padrões de crescimento econômico que têm consequências negativas para o desenvolvimento social, especialmente devido à perseguição desenfreada por lucros e acumulação de riquezas por pequenos grupos que geralmente governam os países. Associado à corrupção elevada, diminuem as possibilidades de que o crescimento econômico possa se refletir no desenvolvimento e melhoria da saúde da população.

O'Langhlin (2012), em seu artigo sobre o desafio da saúde rural publicado na obra Desafios para Moçambique 2012, deixou claro que o argumento de que o crescimento econômico traz o bem-estar das pessoas é desejável, mas, na prática, nem sempre acontece assim. Essa ideia é também confirmada pelo documento do governo Moçambicano, Plano de Ação para a Redução da Pobreza Absoluta 2006 - 2009 (PARPA), ao reconhecer que o crescimento que se registra em Moçambique, nos últimos anos, não se refletiu ainda na melhoria dos indicadores sociais que revelam o desenvolvimento da sociedade. Para além disso, metade da sua população vive em condições indigentes, e uma parte é vulnerável às catástrofes naturais, sobretudo as cheias (MOÇAMBIQUE, 2012), mostrando de forma inequívoca que o crescimento econômico em quase nada acresce o desenvolvimento humano.

O subdesenvolvimento desempenha um papel nefasto na produção de doenças para a população, e, consequentemente, um grave problema sanitário para a população. Ao contrário, quando o desenvolvimento é equitativo entre 
as populações, também se espera a melhoria da vida das pessoas por meio de acesso à água potável, habitação condigna e supressão de outras necessidades básicas. Espera-se, ainda, que haja mais participação ativa da população no processo do desenvolvimento e da melhor distribuição dos bens que daí advém. Nesse contexto, Gadelha (2007) afirma que a saúde constitui uma condição de cidadania, parte inerente ao próprio desenvolvimento, não havendo país no mundo que possa ser considerado desenvolvido com saúde precária.

\section{A pobreza como entrave para o desenvolvimento e saúde da população}

O conceito de pobreza é polissêmico e multidimensional. Desse modo, esta reflexão adota a definição apresentada pelas Naçôes Unidas: impossibilidade de uma pessoa viver com 1,25 dólares americanos por dia para a sua sustentabilidade (pobreza extrema) (UN, 2015). Embora seja um conceito baseado na perspectiva econômica, é de extrema importância, porque a pobreza, em geral e na sua dimensão extrema em particular, está conectada com a má qualidade da saúde da população, fruto da desigualdade social. Silva (2002) vai além, ao mostrar que a pobreza está relacionada ao stress, a mais possibilidade de ter doenças e a dificuldades de acesso às unidades sanitárias. Essa perspectiva de relacionar a pobreza à saúde também é apresentada por Narayan et al. (2000), na obra The voices of the poor: can anyone hear us?, publicada pelo Banco Mundial. Nela, os autores descrevem que a pobreza vai para além de não se ter bens e atinge as esferas psicológica (tristeza, humilhação, vergonha e medo), social (exclusão, falta de convívio) e biológica (vulnerabilidade a doenças). Sinaliza-se também que a pobreza geralmente é associada a outros determinantes de saúde não resolvidos, nomeadamente, falta de habitação condigna; saneamento; alimentação, emprego, lazer e participação no poder, estando a pessoa confinada a condições de vida precárias (WHO, 2010; AHNQUIST; WAMALA; LINDSTRONJ, 2012; GEIB, 2012; AWALLI, 2014; MAXWELL et al., 2015). Essa relação entre saúde e precariedade das condições foi abordada por Akerman et al. (2012), que defendem que, desde os anos 1800, ela já tinha sido relatada por Engels, ao estudar as condições da classe operária na Inglaterra, mostrando claramente a relação entre suas condições de vida e a ocorrência das doenças. 
No início deste milênio, a questão da relação entre saúde e desenvolvimento foi explicitada no contexto dos oito Objetivos de Desenvolvimento do Milênio (ODM), uma vez que, deles, três estão diretamente relacionados à saúde: reduzir a mortalidade infantil, melhorar a saúde materna e combater o HIV/Aids, a malária e outras doenças. Esses objetivos, por si só, não poderão ser atingidos se não estiverem em comunhão com outros, como a erradicação da pobreza e da fome, a educação universal e uma parceria global em prol do desenvolvimento.

Nesses objetivos, revela-se um reconhecimento pela comunidade internacional de que não há saúde sem o desenvolvimento, nem este sem aquela. Ainda, a Declaração do Rio sobre Determinantes de Saúde ressalta que saúde e desenvolvimento devem estar relacionados, enfatizando que deve haver uma melhor governança nesses campos dentro e entre os Estados (WHO, 2011). Contudo, essa posição, enfatizando a inter-relação entre saúde e desenvolvimento, já havia sido afirmada pela mesma OMS quando se criou a Comissão para os Determinantes Sociais e Ambientais de Saúde (WHO, 2004).

Appleton (2000) defendeu que o investimento em capital humano tem mostrado uma contribuição significativa no crescimento econômico e, por outro lado, que o investimento na educação melhora a saúde da população. $\mathrm{Na}$ mesma linha de ideias, Akerman et al. (2012) argumentam que, no processo de saúde e desenvolvimento, deve-se criar condições para que os serviços de saúde possam contribuir para o desenvolvimento inclusivo e sustentável, e isso requer a criação de novas organizações de saúde e profundas mudanças na gestão de serviços de saúde.

\section{A política de combate à pobreza em Moçambique}

O combate à pobreza tornou-se mais vigoroso em 2000 em um dos ODM, erradicar a pobreza. Nele, está implícita e explicitamente reconhecido que o maior entrave do desenvolvimento humano e da boa qualidade da saúde está relacionado à pobreza e que, por isso, esta precisa ser eliminada. Foi baseando-se nos ODM que o Governo da República de Moçambique elaborou a política de combate à pobreza PARPA, que vem sendo atualizada. Por exemplo, o PARPA II inclui nove assuntos transversais que estão relacionados à pobreza absoluta, nomeadamente: assuntos de gênero; HIV/Aids; segurança alimentar e nutrição; ciência e tecnologia; desenvolvimento rural, calamidades e desminagem. $\mathrm{Na}$ política, o governo reconhece que o HIV afeta negativamente a economia de 
Moçambique, porque reduz o crescimento da população humana, além de reduzir a acumulação do capital físico e da produtividade da força do trabalho.

Todavia, a luta pela erradicação da pobreza, declarada pelo Estado, ainda não passou do papel para as açôes concretas. É notório o ceticismo patente no Relatório sobre os ODM, elaborado pelo Ministério de Planificação e Desenvolvimento de Moçambique, ao declarar que o país dificilmente poderá atingir a redução de dois terços a taxa de mortalidade abaixo de cinco anos, em três quartos a taxa de mortalidade materna e de travar o alastramento de HIV/Aids, malária e outras doenças (MOÇAMBIQUE, 2011). Fazem parte dos constrangimentos de alcance das metas do ODM o crescimento com taxas baixas ou nulas da produtividade agrícola e trocas. Além disso, a população moçambicana deparouse com disponibilidades limitadas de alimentos de produção própria e custos muito altos de alimentos importados dos mercados internacionais, uma vez que o país não produz o suficiente para a sua população. Observou-se, ainda, o aumento nos custos dos combustíveis, que tornou substancialmente mais cara a distribuição das importações e o transporte dos locais de excesso produtivo para áreas de déficit (MOÇAMBIQUE, 2011).

Assim, a pobreza em Moçambique tornou-se um ciclo vicioso entre as populações das áreas rurais, onde faltam água potável, alimentação, moradia condigna e infraestrutura sanitária adequada, para além de haver frágeis políticas de mobilização das comunidades em usar os serviços sanitários em locais onde eles existem. Shenga, Pedro e Muendane (2013) declararam que a pobreza em Moçambique está cada vez mais enraizada nas zonas rurais. Essa relação entre cidades e zonas rurais já tinha sido constatada por Silva (2002), ao falar de pobreza e saúde no Brasil, descrevendo claramente que as diferenças entre as cidades e regiōes rurais é notória, tendo estas maiores problemas de saúde e dificuldades de desfrutar de uma saúde de qualidade em relação às cidades.

Nesse contexto, nos indagamos: quais são as políticas de saúde existentes em Moçambique? O SNS de Moçambique tem tido políticas pouco abrangentes, e algumas delas ineficazes, apresentando dificuldades de pessoal e sem financiamento. Tais são os casos de política e estratégia de saúde sexual reprodutiva de adolescentes; política de combate à tuberculose e controle de infecções e política nacional de saúde neonatal infantil. Esses exemplos caracterizam-se por serem focais e não terem reduzido significativamente os problemas que se propuseram resolver. Aliás, alguns desses problemas estão em crescimento, 
como é o caso da tuberculose, cujo número de infectados em 2014 foi de 58.270, numa população de 27.216.000 habitantes, representando um aumento de $26 \%$, quando comparado aos casos de 2010. Ainda, o aumento anual nos últimos anos foi, em média, de 6\%, mas, em 2014, atingiu 9\% (MOÇAMBIQUE, 2015; WHO, 2015). Portanto, tanto o combate à pobreza absoluta como as políticas de saúde ainda não conseguem ser eficazes no país.

As iniquidades regionais no acesso à saúde são claras nas regiões Norte e Centro de Moçambique, com maior número da população que possui menores condições de acesso aos médicos, em termos de proporção, e menor número de hospitais (MOÇAMBIQUE, 2015), sendo nessas regiōes que a saúde e o desenvolvimento são mais críticos.

\section{Compreendendo as consequências da desconexão entre saúde e desenvolvimento em Moçambique}

Em Moçambique, não é tão difícil descobrir que saúde e o desenvolvimento estão desconectados: basta verificar que $43 \%$ das crianças menores de 5 anos sofrem de subnutrição crônica e 6\% sofrem de subnutrição aguda (MOÇAMBIQUE, 2011). Portanto, aproximadamente $50 \%$ das crianças moçambicanas são malnutridas, como resultado direto da falta de alimentação e do subdesenvolvimento. O’Langhlin (2012) e WHO (2012) já defenderam que o estado nutricional reflete a qualidade de vida da população no seu todo ou de um determinado segmento populacional, e ainda argumentaram que as crianças das áreas rurais são as mais subnutridas se comparadas às das áreas urbanas.

Outro exemplo da desconexão entre saúde e desenvolvimento vem da Avaliação Nacional de Necessidades em Saúde Materna e Neonatal em Moçambique 2007/2008, que revela as principais causas de morte neonatal institucional: a prematuridade (50\%), asfixia grave (32\%) e a sepse neonatal (29\%). Esses dados demonstram as consequências de uma saúde materna deficiente, de cuidados inadequados durante a gravidez e os primeiros dias após o parto, além da necessidade urgente de aumentar a cobertura de atenção ao parto institucional, os cuidados essenciais ao recém-nascido e a cobertura da consulta até o sétimo dia (MOÇAMBIQUE, 2010). Por outro lado, revelam a falta de êxito das políticas de saúde existentes em Moçambique. Nesses casos, é necessário, como defendem Vilana e Elias (2007), que haja a combinação virtuosa entre o desenvolvimento 
econômico e social, capaz de melhorar a saúde da população. A não observância ou reconhecimento dessa desconexão acarreta na manutenção do status quo e na valorização das condições de pontual do crescimento de vida, em detrimento da qualidade de vida dos moçambicanos, apesar de a saúde ter sido reconhecida como direito humano pela comunidade internacional.

\section{Direito à saúde em Moçambique}

O direito à saúde remonta ao movimento de Saúde Pública do século XIV, com a criação da Nações Unidas, onde esse passa a ser afirmado no contexto do sistema internacional de direitos humanos, especialmente após a Declaração Universal dos Direitos Humanos de 1948 (ZAIDEI, 2012). Em 1978, com a Conferência Internacional sobre Cuidados de Saúde que ocorreu na cidade de Alma Ata, na Antiga URSS (União das Repúblicas Socialistas soviéticas), pontuou-se que "uma das principais metas sociais dos governos, das organizaçóes internacionais e de toda a comunidade na próxima década deve ser a de que todos os povos do mundo até o ano de 2000 atinjam um nível de saúde que lhes permita levar uma vida social e economicamente satisfatória”. Dessa forma, direta ou indiretamente, essa declaração reconhece que o estado de direito à saúde é de todo ser humano, independentemente da sua raça, cor, credo, religião e nacionalidade. Todavia, o direito à saúde reafirmado pela Declaração de Alma Ata ainda, três décadas depois, continua a ser um desejo para os países da África Subsaariana e, principalmente, para a Moçambique.

A Carta Africana dos Direitos Humanos e dos Povos, aprovada pela Conferência Ministerial de Organização da Unidade Africana (OUA) em Banjul, Gâmbia, em 1981, no seu artigo 16, defende que todas as pessoas têm direito a gozo do melhor estado de saúde física e mental que forem capazes de atingir. Esse documento torna claro o reconhecimento do direito à saúde nos países africanos. Nessa perspectiva, a própria Constituição da República de Moçambique, de 2004, preconiza que a saúde é um direito e garante que todos os cidadãos têm o direito à assistência médica, além de consagrar que todos gozam dos mesmos direitos. Contudo, apesar de todo esse arcabouço jurídico, cerca de $50 \%$ da população continua a não ser assistida pelo SNS, o que mostra que, embora Moçambique tenha ratificado a Carta Africana e a Constituição sublinhe o direito à saúde, o país ainda não conseguiu implementá-lo. 


\section{Considerações finais}

Este artigo abordou a relação existente entre saúde e desenvolvimento na África Subsaariana, especificamente em Moçambique, um país que, por um lado, apresenta um crescimento econômico importante, mas, por outro, onde as condiçõoes de vida e de saúde das populações são cada vez mais precárias e a melhoria de sua saúde continua a ser uma meta difícil de ser atingida. Além disso, o país apresenta desigualdades sociais acentuadas entre as zonas rurais e as cidades e entre os ricos e os pobres.

O fenômeno da pobreza é paradoxal e torna a sua ligação evidente com a precária saúde da população moçambicana, que refuta a ideia de que a pobreza se origina no próprio homem e a sua eliminação depende da sua vontade. É necessário, sim, um Estado forte capaz de identificar as causas e estratégias para combatê-la desenhando políticas e ações concretas.

A falta do desenvolvimento em saúde em Moçambique é caracterizada, na população, pela má qualidade de vida e pela falta de educação básica capaz de levar a população a reconhecer minimamente medidas para prevenir determinadas doenças e como tratá-las, apelando, dessa forma, para uma reflexão profunda e uma atitude de mudanças rápidas na área de educação e saúde, incluindo a agricultura.

Em Moçambique, o desenvolvimento humano ainda está em situação não aceitável, impelindo, para isso, uma rápida conexão entre a saúde e desenvolvimento, vigilância e leituras constantes da evolução do mundo em matéria desses campos, integrando políticas de boas práticas usadas por outros países, cuja situação já foi semelhante à de Moçambique.

O país, por estar, aos poucos, entrando no sistema de industrialização, requer políticas de atenção em saúde e de redistribuição da renda. A não incorporação dos aspectos da saúde no desenvolvimento poderá aumentar ainda mais o fosso de desigualdades sociais entre os ricos e pobres, ao passo que a melhoria das condiçôes de vida e de saúde poderá refletir-se na melhoria da qualidade de vida da população. Uma população doente implica uma nação também com deficiência na sua evolução, o que requer maior atenção em setores-chave (saúde, educação), que, coordenados, melhoram o desenvolvimento e a saúde das pessoas.

Um equilíbrio entre saúde e desenvolvimento revela maior bem-estar da população e melhor confiança em investimentos, para além de diminuir mortes 
desnecessárias em indivíduos das primeiras faixas etárias. O desenvolvimento de uma sociedade e de um Estado proativo faz com que as pessoas atinjam a autonomia de pensar, de fazer escolhas e ter consciência da importância de preservar a própria saúde e a dos outros, exercendo o seu próprio direito à saúde ${ }^{1}$.

\section{Referências}

AHNQUIST, J.; WAMALA, S.; LINDSTROM, M. Social determinants of health - A question of social or economic capital? Interaction effects of socioeconomic factors on health outcomes. Social Science \& Medicine, n. 74, p. 930-939, 2012.

AKERMAN, M. et al. Saúde e desenvolvimento: que conexões? In: CAMPOS, G.W S. (Org.). Tratado de saúde coletiva. 2. ed. Revista e aumentada. São Paulo: Hucitec, 2012. p.123-147.

ALMEIDA-SANTOS, A.; ROFFARELLO, L.M.; FILIPE, M. Moçambique 2014. Moçambique: OECD, 2014. Disponível em: <http://www.africaneconomicoutlook.org/ fileadmin/uploads/aeo/2014/PDF/CN_Long_PT/Mozambique_PT_BAT.pdf>. Acesso em: 24 dez. 2015.

ANDRADE, E.O et al. Índice de desenvolvimento em saúde: conceituação e reflexões sobre sua necessidade. Rev. Assoc. Méd. Bras. Brasília, v. 58, n. 4, p. 413-421, 2012.

APPLETON, S. Education and Health at the household level in sub-Saharan Africa CID working paper- working papers: Center for international Development at Harvard University, n. 33, jan. 2000. p. 18-40.

APPLETON, S.; HODDINOTT, J.; MACKINNON, A. Education and health in SubSaharan Africa. Journal of International Development, v. 8, n. 3, p. 307-339, 1996.

AWALLI, J. O. Poverty and health care demand in Kenya. BMC Health Services Research, n. 14 , p. 5650-5657, 2014.

BANCO MUNDIAL. O relatório sobre o desenvolvimento em Moçambique. Maputo: Banco Mundial, 2013.

CARTA AFRICANA dos Direitos Humanos e dos povos. Abuja - Gâmbia 1981. Disponível em: <http://www.dhnet,org.br/discidos/sip/africa/banjul.htm>. Acesso em: 09 set. 2013.

FURTADO, C. Introdução ao desenvolvimento: enfoque histórico estrutural. 3a edição. São Paulo: Paz e Terra, 2000. 49p.

GADELHA, C.A.G. Desenvolvimento e saúde: em busca de uma nova utopia. Saúde em Debate. Rio de Janeiro, v. 19, n. 71, p.326-327, 2007.

GADELHA, C.A.G. et al. Saúde e territorialização na perspetiva do desenvolvimento. Ciência \& Saúde Coletiva, Rio de Janeiro, v. 16, n. 6, p. 3003-3016, 2011. 
GEIB, L.T.C. Determinantes sociais do idoso. Ciência \& Saúde Coletiva, v. 17, n. 1, p. 123133, 2012.

LAU, C.; MUULA, A.S. HIV/AIDS in sub-saharian Africa. Croatian Medical Journal, v. 45, n. 4, p. 402-414, 2004.

MAXWELL, A.E.; et al. Social determinants of health in the Mixtec and Zapotec community in Ventura Country, California. International Journal for equity in Health, v. 14, n. 16, 2015. Disponível em: <http://www.equityhealthj.com/content/14/1/16>. Acesso em: 03 jan. 2016.

MOÇAMBIQUE. Constituição da República. Maputo, 2004. 97p.

. Inquérito Demográfico e de Saúde 2011. Maputo: Instituto Nacional de Estatística/ Ministério da Saúde, 2011, 430p.

. Plano de Acção Para a Redução da Pobreza Absoluta. 2006-2009. Maputo, 2006. 46p. . Relatório sobre os Objetivos de Desenvolvimento de Milênio. Maputo: Ministério da Planificação e Desenvolvimento, 2012. 25p.

. Relatórios das atividades desenvolvidas durante o ano de 2014. Maputo: Ministério da Saúde, 2015. 57p.

- Relatório sobre os objectivos do Desenvolvimento de Milênio de 2010. Maputo: Ministério da Saúde, 2011. 20p.

NARAYAN, D. et al. Voices of the poor. Can anyone hear us? Oxford: Oxford Press for the World Bank, 2000. 280p.

O’LANGHLIN, O. O desafio de saúde Rural. In: BRITO, L. et al. Desafios para Moçambique 2012. Maputo: IES, 2012. p. 333-370.

OMS. Redução das desigualdades no periodo de uma geração. Igualdade na saúde através de acção sobre os determinantes sociais. Relatório final da comissão para os determinantes sociais de saúde. Lisboa: OMS, 2010. 276p.

- Sistemas de saúde em África - percepções e perspectivas das comunidades multipaíses, OMS: Escritório Regional para África, 2012. 111p.

PNUD. Human Development Report 2015: work for human development. New York: PNUD, 2015. 286p.

SHENGA, C., PEDRO, C.; MUENDANE, E. Perspectiva Multidimensional da Experiência da Pobreza em Moçambique, 2002-2012. Afrobarometer, 2013. Disponível em: <http:// www.cpgd.org.mz/sites/cpgd.org.mz/files/publications/ABBP1_Pobreza10August2013. pdf>. Acesso em: 16 set 2014.

SILVA, T. M. A pobreza e saúde: que perspectiva de vida saudável teremos para o século XXI? In: CIANCIARULLO, T.I.; CORNETTA, T. Saúde, desenvolvimento e globalização. São Paulo: Ícone, 2002. p. 37-53. 
TISCH, S.; WALLACE, M. Dilemmas of development assistance: the why, and who of foreign Aid. San Francisco: Westview Press, 1994. 180p.

UN. The Millennium Development goals Report. New York: UN, 2015. 75p.

VILANA, A. L. D.; ELIAS, P. E. M. Saúde e Desenvolvimento. Ciência \& Saúde Coletiva, v. 12, n. sup, p. 1765-1777, 2007.

WHO. Declaração política do Rio sobre determinantes sociais da saúde. Rio de Janeiro, Brasília, 2011. Disponível em: <http://www.who.int/sdhconference/declaration/Rio_political_ declaration_portuguese.pdf>. Acesso em: 27 dez. 2015.

. Global tuberculosis report 2015., Geneva, Switserland: WHO, 2015. 204p. . Global tuberculosis control. Genebra: WHO, 2006. 50p.

WILSON-STRYDOM, M.; FONGWA, S. N. Um perfil do ensino superior na África Austral. Johannesburg: SARUA, 2012. 107p.

WORLD BANK. Global Economic Prospects: Managing growth in a volatile world. Washington, DC, 2012. 150p.

ZAIDI, S. L. Objetivo 6 do Desenvolvimento do Milênio e de Direitos à saúde: contradições ou complementares? Revista internacional de direitos humanos, v. 7, n. 7, p. 129-149, 2012.

\section{Nota}

${ }^{1}$ Os autores declaram ter participado da concepção redação do artigo, revisão crítica relevante do conteúdo intelectual e aprovação final da versão a ser publicada do artigo. F. Mitano trouxe a reflexão do seu país ao mobilizar textos que reportam a realidade de Moçambique para a análise. C. Ventura contribuiu com o arcabouço teórico de desenvolvimento e pobreza. P. F. Palha contribuiu com a reflexão das informações da OMS, Nações Unidas e o Banco Mundial sobre os países em desenvolvimento. 
Health and development in Sub-Saharan Africa: a reflection focusing on Mozambique The recognition that population health improvement is linked to the advancement of socioeconomic conditions is visible in the last years, stimulating changes in national and international policies. However, in several countries from Sub-Saharan Africa, if not in all of them, this relationship is inversely proportional. This article aims at reflecting about the connection between health and development in Mozambique. This reflection offers a comprehension about the economic growth registered in the country, with the great majority of the population living in precarious conditions and with a multiplicity of evitable diseases, demonstrating that the adopted policies do not favor an improvement of health and development. This situation requires the country to apply policies of income redistribution and health care to the most needed population.

Key words: health; development; poverty. 